\title{
Relation between bone density and primary implant stability
}

\author{
Núria Farré-Pagès ${ }^{1}, \mathrm{M}^{\mathrm{a}}$ Luisa Augé-Castro ${ }^{2}$, Fernando Alaejos-Algarra ${ }^{3}$, Javier Mareque-Bueno ${ }^{4}$, Eduard \\ Ferrés-Padró ${ }^{5}$, Federico Hernández-Alfaro ${ }^{6}$
}

\author{
${ }^{1}$ DDS, Master in Oral Implantology, PhD student, Medicine, Surgery and Oral Implantology Department, Dental School. Inter- \\ national University of Catalonia \\ ${ }^{2}$ MD, DDS, Master in Oral Implantology, A. Professor, Medicine, Surgery and Oral Implantology Department, Dental School. \\ International University of Catalonia \\ ${ }^{3}$ MD, DDS, PhD Master in Oral Implantology, A. Professor, Medicine, Surgery and Oral Implantology Department, Dental \\ School. International University of Catalonia \\ ${ }^{4}$ MD, DDS, PhD OMFS, A. Professor, Medicine, Surgery and Oral Implantology Department, Dental School. International \\ University of Catalonia \\ ${ }^{5}$ MD, DDS, PhD OMFS, Prof. and Head of Department, Medicine, Surgery and Oral Implantology Department, Dental School. \\ International University of Catalonia \\ ${ }^{6}$ MD, DDS, PhD OMFS, Prof. and Director Master in Oral Implantology, Medicine, Surgery and Oral Implantology Department, \\ Dental School. International University of Catalonia
}

Correspondence:

Medicine, Surgery and Oral Implantology Department,

Faculty of Dentistry. Universitat Internacional de Catalunya,

Campus Sant Cugat,

08195 Sant Cugat del Vallès,

Barcelona. Spain

nuriafarrepages@telefonica.net

Farré-Pagés N, Augé-Castro ML, Alaejos-Algarra F, Mareque-Bueno J, Ferrés-Padró E, Hernández-Alfaro F. Relation between bone density and primary implant stability. Med Oral Patol Oral Cir Bucal. 2011 Jan 1;16 (1):e62-7.

http://www.medicinaoral.com/medoralfree01/v16i1/medoralv16ilp62.pdf

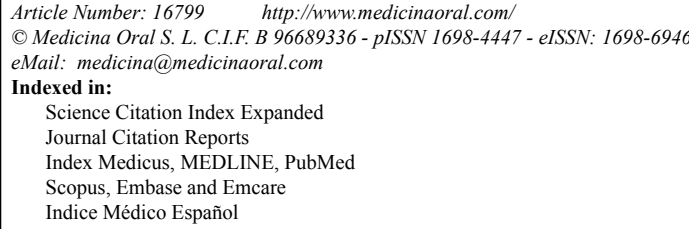

\begin{abstract}
Aims: This study aims to relate bone density in Hounsfield units $(\mathrm{Hu})$ with the primary implant stability measured by insertion torque $(\mathrm{Ncm})$ and resonance frequency analysis (ISQ).

Materials and Methods: Ten patients were included in this study. A total of 54 implant sites were provided from 10 computerized tomography scans. The computerized tomography scan was used for the preoperative evaluation of bone density for each patient. The bone mean density around planned implants was determined with Physioplanet TM software. Bone quality according resistance to drilling, insertion torque and resonance frequency measurements were recorded.

Results: A statistically significant relationship was observed between bone quality density and location with ISQ values.

Conclusions: This research demonstrates a strong relationship between the bone density values from computerized tomography and the location in the maxillaries. A correlation exists between bone quality, according to the Lekholm \& Zarb classification, and Hu computerized tomography values. The primary implant stability measured with resonance frequency analysis depends on bone density values, bone quality and implant location.
\end{abstract}

Key words: Hounsfield unit, bone quality, implant stability, computerized tomography (CT scan), dental im- 


\section{Introduction}

Currently the use of osseointegrated implants to treat partially or completely the edentulous arch is considered reliable and predictable, with a success rate of $98 \%$ or higher (1). Implant success depends on patient characteristics, surgical technique and implant design. Factors such as bone quality and quantity determine the procedure and the type of implant according to their design and surface treatment.

To achieve a good result with our implant treatment, a thorough diagnosis is essential, starting with the patient medical history, an analysis of the occlusion, a waxing and a complete diagnostic using panoramic radiography and computed tomography (CT). Panoramic radiography will give an overview of the anatomical structures of the jaw. And the CT scan offers more specific data such as height, width or bone density in the peri-implant area.

Bone density is a key factor to take into account when predicting implant stability. A good surgical technique and good stability favors implant osseointegration (2). Clinical studies show greater implant survival in the mandible than in the upper maxilla, due to the area's characteristics. This survival is limited by bone quality, i.e. bone density (3).

Several studies in the literature corroborate a higher failure rate of implants placed in type IV bone. Likewise, we found good results with implants placed in type I, II, and III bone, the latter being the optimal type of bone for adequate implant stability. Bone density and implant stability are important factors for implant osseointegration, which has been widely demonstrated by several authors (4).

Martinez et al. (5) assessed how to achieve optimal primary implant stability in unfavorable bone density. In poor bone density, primary implant stability is lower than on implants placed in denser bone, but the secondary stability is similar in different types of density. Stability is also influenced by the surgical technique, the surface implant morphology, or its diameter, bone compaction techniques and cortical anchor for implant placement. Wide diameters that increase the contact area between the treated bone and implant surface treated will increase primary stability. Anatomically, tapered implants have more primary stability than cylindrical implants, just as they have a greater diameter in the crestal portion (5).

This study aims to relate bone density in Hounsfield units $(\mathrm{Hu})$ with primary implant stability measured by insertion torque $(\mathrm{Ncm})$ and resonance frequency analysis (ISQ).

\section{Materials and Methods}

The following clinical study is quantitative and qualitative set in a historical context, i.e. we have studied the events as they occurred during implant placement. Ten patients ( 4 females and 6 males, mean age 53 between 38 and 78 years old) whose previous CT scan indicated suitability for implant treatment. The implant insertion was with a partial or total surgical guide. We excluded those who had uncontrolled psychiatric disease, uncontrolled systemic disease, patients in treatment with radio and / or chemotherapy, pregnant patients, clinical post-implant removal, clinical implants placed in sinus lift prior and clinical implants placed in regenerated ridges.

\section{Pre-operative bone density evaluation (Hu)}

A preoperative CT scan was used to evaluate bone density for each patient. With the software Physioplanet ${ }^{\mathrm{TM}}$ mean bone density around planned implants was determined. This software is a digital display of DICOM images from the $\mathrm{CT}$ scan with functions to determine dimensions, and planning of dental implants and surgery with or without surgical guidance such as bone density and quantity. The software has the ability to determine bone density from a conventional tomographic image of linear beam. The method is based on direct measurement of the gray scale image of the CT scan, which relate to the linearity of $\mathrm{Hu}$, so that optical density at a point is correlated with its bone density. Bone density values varied from $84 \mathrm{Hu}$ in the posterior maxilla to $1327 \mathrm{Hu}$ in the anterior mandible region. Mean mandibular density was higher than mean maxilla density, with $776 \mathrm{Hu}$ in anterior region, ranging from $325 \mathrm{Hu}$ and $1327 \mathrm{Hu}$. $746.727 \mathrm{Hu}$ in posterior mandible region ranging from $279 \mathrm{Hu}$ to $1016 \mathrm{Hu}, 431.6 \mathrm{Hu}$ in anterior maxilla region with a range from $101 \mathrm{Hu}$ to $595 \mathrm{Hu}$, and $193.286 \mathrm{Hu}$ in posterior maxilla with a range from $84 \mathrm{Hu}$ to $430 \mathrm{Hu}$.

\section{Surgical procedures}

Each patient signed a treatment consent letter and followed a pre-surgical and surgical protocol:

Oral hygiene two weeks before surgery, in case with plaque index $>20 \%$. Chlorhexidine $(0.12 \%)$ rinses $2 /$ day during $45 \mathrm{sec}$., from three days before surgery. Antibiotic therapy was given to the patients: amoxicillin $2 \mathrm{~g} \mathrm{VO}$ an hour before the treatment and $500 \mathrm{mg} / 8 \mathrm{~h}$ during 6 following days. Any penicillin allergy was treated with clindamycin $600 \mathrm{mg}$ an hour before the surgery and $300 \mathrm{mg} / 6 \mathrm{~h}$ during the 5 following days. Local anesthesia was used in all cases: articaine $40 \mathrm{mg} / 0.01 \mathrm{mg}$ epinephrine.

\section{Bone quality according resistance to drilling}

Implants were placed according to the standard manufacturer systems protocol, completely or partially guided surgical templates were used to place them in the intended regions. Resistance was recorded of the implant bed milling classified as type I, II, III, IV according to the classification of Lekholm \& Zarb (1985).

Insertion torque

At the moment of the implant placement, initial inser- 
tion torque of $20 \mathrm{Ncm}$ was established, increasing in $5 \mathrm{Ncm}$ until total implant of the insertion. The maxim insertion torque was $50 \mathrm{Ncm}$, implant stability data was collected through the insertion torque.

Resonance frequency analysis (RFA)

Resonance frequency measurements were recorded using Osstell ${ }^{\mathrm{TM}}$ mentor. It was necessary to screw a disposable magnetic attachment (Smartpeg ${ }^{\text {TM}}$ ) to implant 4-5 Ncm. Magnetic attachment cannot have any contact with any metallic instrument before it is screwed. Smartpegs ${ }^{\mathrm{TM}}$ compatible with diameter $3.5 \mathrm{~mm}, 4 \mathrm{~mm}$, $4.5 \mathrm{~mm}$ Astra $^{\circledR}$ system connection; diameter $4.2 \mathrm{~mm}$, $3.75 \mathrm{~mm} \mathrm{Mis}{ }^{\circledR}$ (Seven and Biocom) system connection was used. Each implant was measured twice from two different angles, around 90 degrees and parallel to the crestal line. After analyzing the primary stability of each implant, the Smartpeg ${ }^{\mathrm{TM}}$ was removed and the surgical incision area was sutured with a monofilament suture 4/0. To prevent early postoperative complications, antibiotics and analgesics were prescribed and the patient given information on postoperative care. The suture was removed one week after surgery.

\section{Collection data}

A total of 54 implants were inserted. There were 25 Astra $^{\circledR}, 22$ Mis-Seven $^{\circledR}$, and 7 Mis-Biocom ${ }^{\circledR}$ dental implants. Of the 54 implants, 30 were cylindrical in design, the others were conical. The cutting protocol accorded to each implant system. 24 implants were placed in maxilla, 15 in the anterior region and 9 in the posterior region. 30 implants were placed in the mandible region,
19 implants in anterior mandible region and 11 in the posterior of mandible. All of them were of the internal connection system type with a length between $8 \mathrm{~mm}$ and $13 \mathrm{~mm}$ and a diameter of $3.5 \mathrm{~mm}$ and $5 \mathrm{~mm}$.

All data collected were unified in a same Excel table. Statgraphics Plus 5.1 statistical software program was used to compare data analysis. We related the implant location with bone density ( $\mathrm{Hu}$ ) and with stability. We also evaluated the relationship between bone density $\mathrm{Hu}$ and bone quality (type I, II, III, IV), the primary stability - resonance frequency (ISQ) - and implant insertion torque $(\mathrm{Ncm})$. We determined the nature of each variable and, as the distribution was not normal, we applied the Kruskal-Wallis test to analyse the data. This test works with the average and not with the mean. A simple regression was used to analyse the dependence between quantity variables. Moreover, a new variable was determined to unify variables determining stability by a multivariate method.

\section{Results}

A significant statistical relationship was obtained between the implant location and Hu. Likewise, there was relationship between the location and primary stability in terms of ISQ measurements. Regarding the relationship between bone density in $\mathrm{Hu}$ or bone quality and primary implant stability, there was only a relationship with resonance frequency measurements of implants. Related data with insertion torque were not statistically significant (Table 1).

Table 1. Statistical results.

\begin{tabular}{|l|l|l|l|c|}
\hline \multicolumn{1}{|c|}{ Variables } & Normality & Test & r-value & p-value \\
\hline Location/HU & NO & Kruskal-Wallis & 0.00006 & $<0.05$ \\
\hline Location/Ncm & NO & Kruskal-Wallis & 0.202111 & $>0.05$ \\
\hline Location/ISQ & NO & Kruskal-Wallis & 0.0064 & $<0.05$ \\
\hline HU/Ncm & - & Simple Regression & 0.0859 & $>0.05$ \\
\hline HU/ISQ & - & Simple Regression & 0.0474 & $<0.05$ \\
\hline Bone quality/Ncm & NO & Kruskal-Wallis & 0.388 & $>0.05$ \\
\hline Bone quality /ISQ & NO & Kruskal-Wallis & 0.0364 & $<0.05$ \\
\hline Bone quality /HU & NO & Kruskal-Wallis & $3.1538 \mathrm{E}-7$ & $<0.05$ \\
\hline Ncm/ISQ & - & Simple Regression & 0.0134 & $<0.05$ \\
\hline
\end{tabular}

HU: Hounsfield units, ISQ: Implant stability quotient value. 


\section{Discussion}

Location vs $H U$

In the present study, the anterior mandible region presents higher bone density than the posterior mandible, followed by anterior maxilla and posterior maxilla with lower bone densities. Hence, the lower maxilla presents higher bone density than the upper maxilla.

This data is in agreement with Norton and Gamble (6). However, in their study, they identified a higher mean bone density in the anterior region of the maxilla than in the posterior region of the mandible, $696 \mathrm{Hu}$ and $669 \mathrm{Hu}$ respectively. Whereas, we observed higher density values in the posterior of the mandible than in the anterior of the maxilla, $746.727 \mathrm{Hu}$ and $431.6 \mathrm{Hu}$ respectively. Moreover, we detected a mean density value of $776 \mathrm{Hu}$ in the anterior mandibular region, lower than that described by Norton and Gamble (6) $(970 \mathrm{Hu})$, and even lower than that described by Turkyilmaz et al. (7), who described a bone density value in said region of 994.9Hu. Values are also lower than those described by these authors in the posterior maxilla. This may be due to the patient age of the sample averaging 53 years, and with $83.333 \%$ of implants placed in patients older than the sample average. Given that the older the patient, the greater the decrease of bone density.

Location vs. ISQ and Insertion Torque

Implants placed in the anterior mandibular region present higher primary stability measured by resonance frequency of ISQ values than implants placed in the posterior mandibular or maxillary area. This concept concurs with Morris et al. (8), who described less stability in the maxilla than in mandible, although they measured stability with the Periotest system. Our study measured the stability by RFA (ISQ) and implant insertion torque $(\mathrm{Ncm})$. We found a correlation between location and ISQ values, although our data suggest, that posterior maxilla presents high ISQ values, presumably due to the small number of implants placed in that location. In contrast, in regarding the relationship between location and insertion torque, there were no differences according to location, which is confirmed by Beer et al. (9). This data may be influenced by the amount of cortical or cancellous bone surrounding the implant.

We recorded a higher mean insertion torque in the mandibular than in the maxilla $(42.34 \mathrm{Ncm}$ and $40.22 \mathrm{Ncm}$ respectively). Similarly, we found a higher mean insertion torque in the anterior region $(42.22 \mathrm{Ncm})$ than in the posterior region $(40 \mathrm{Ncm})$. Turkyilmaz et al. (10), also defined higher insertion torque values in the jaw area than in the maxillary, with an average insertion torque of $40.5 \mathrm{Ncm}$ and $37.3 \mathrm{Ncm}$, respectively. Moreover they recorded a higher insertion torque in the anterior region than in the posterior region, both in the maxillary. Although no statistically significant differences were recorded between insertion torque and the implant sites, whether mandibular or maxillary, they did report statistically significant differences between the insertion torque and the area, depending on whether it is the anterior or posterior of the maxilla. Contrary to their results, we found no statistically significant differences according to the anterior or posterior and mandibular or maxillary area. We observed only a slight trend, with a clinical difference.

\section{$H U$ vs ISQ and Insertion Torque}

Stability varied according to the location. We obtained more primary implant stability in areas with greater bone density in the $\mathrm{CT}(\mathrm{Hu})$, such as in the anterior and posterior mandibular region. Thus, the greater the $\mathrm{Hu}$ value, the greater is the primary stability measured in ISQ values. Turkyilmaz et al. (11) referred in 2006, to similar data about the relationship between $\mathrm{Hu}$ and ISQ values as well as the relationship between insertion torque and agreement with Ikumi and Tsutsumi (12). These results suggested bone density by $\mathrm{Hu}$ as a method to predict primary stability. Friberg et al.(13) and da Cunha et al.(14) are in agreement with the Turkyilmaz et al. (10) and Ikumi and Tsutsumi (12) results, finding a significant relationship between insertion torque and ISQ values, which coincides with our results. Contrary to Beer et al. (9) no direct relationship was found between $\mathrm{Hu}$ and primary stability in terms of implant insertion torque. Ikumi and Tsutsumi (12), use the same system to register the insertion torque, finding a statistically significant relationship between the insertion torque and bone density measured in Hu. Unlike our results, which coincide with Ikumi and Tsutsumi (12), Turkyilmaz et al. (10) obtained similar results in their study regarding the relationship between $\mathrm{Hu}$ and insertion torque.

We attributed our findings to the method used to collect the insertion torque data. Ikumi and Tsutsumi (12), use OsseoCare ${ }^{\circledR}$ (Nobel Biocare AB, Göteborg, Sweden) device in his study. While in our study, we used different implant devices. The insertion torque was recorded only in the final insertion torque of the total implant insertion. Moreover, the implants that were inserted in their final phase with a ratchet device were recorded as a $50 \mathrm{Ncm}$ torque, which in reality was higher.

Bone quality vs ISQ and Insertion Torque

A statistically significant relationship between different bone qualities according to Lekholm \& Zarb classification and between the localization of the implant and the implant stability, measured with ISQ values, was found. The bone quality type I have greater ISQ values than the type II, III and IV. This finding is in agreement with the Huang et al. (15) published study, which describes that bone quality conditions primary stability. While, in our study the relationship between the level of bone quality and primary stability in terms of insertion torque was analyzed and there was found no statistically significant 
differences. Furthermore, Friberg et al. (16) report a relationship between different bone qualities, insertion torque and resonance frequency measurements.

The results of our study may reflect the same reason why we found no relationship between $\mathrm{Hu}$ and insertion torque and can be attributed to the method used to record the insertion torque.

\section{Bone quality vs $H U$}

On the relationship between bone quality according Lekholm \& Zarb classification and bone density values $\mathrm{(Hu}$, there was a significant statistical relationship, with clearly different $\mathrm{Hu}$ values intervals for each type of bone, according with Norton and Gamble (6), Shahlaie et al. (17) y Aranyarachkul et al. (18) results. Although Shahlaie et al. (17) determine bone quality, according to Lekholm \& Zarb classification was originally based on a radiographic evaluation. After, the same classification was applied to the cutting resistance value from tactile sensation.

The implant insertions in our study were carried out by different surgeons with a similar level of experience. Each surgeon scored the bone quality in a subjective way. Despite this aspect, and the data quality after analysis, there is a strong relationship between bone quality and $\mathrm{Hu}$. Various clinicians collected data except bone density $(\mathrm{Hu})$. This fact does not represent a limitation since clinicians had the same experience level in implantology practice. Clinicians were able to determine bone quality as cutting torque and they were not influenced by previous studies of bone density as $\mathrm{Hu}$. Hu data was assessed by a secondary researcher. However there are other studies that describe the inability of the clinician to detect bone quality. Trisi and Rao (19) evaluate the ability of the surgeon to distinguish the type of bone quality from the resistance to drilling. He concluded that clinicians cannot differentiate between bone quality type III as type II, only between type I and type IV.

\section{Insertion Torque vs ISQ}

It was observed that the correlation between primary implant stability values ISQ and Ncm was statistically significant, as described by Turkyilmaz et al. (10). On the contrary Friberg et al. $(13,16)$ and da Cunha et al. (14) reported no relationship between insertion torque and ISQ values, probably due to the two difference types of implants that were used. In our study three different implants were used, Astra ${ }^{\circledR}$, Seven-Mis $^{\circledR}$, Biocom-Mis $^{\circledR}$, in two different groups, conical and cylindrical design. The different systems, the small sample and the use of different implants devices could affect the relation between insertion torques $(\mathrm{Ncm})$ and implant stability (ISQ). There is a statistically significant correlation between the two parameters, but not strong.

\section{Conclusion}

We conclude that, the present study demonstrates the relationship between the bone density values from computerized tomography $(\mathrm{Hu})$, located in the maxillaries, and bone quality by Lekholm \& Zarb classification.

The primary implant stability measured with resonance frequency analysis (ISQ) depends on bone density values, bone quality and implant location. Implants inserted in location with higher bone density have more stability, but we cannot predict the implant insertion torque based on the bone density values $(\mathrm{Hu})$ and the implant location.

Finally, with higher bone density values $(\mathrm{Hu})$ and higher primary implant stability measured in ISQ values, Hounsfield units can be used as a diagnostic parameter to predict possible implant stability.

\section{References $\quad$ References with links to Crossref - DOI}

1. Jemt T, Lekholm U, Adell R. Osseointegrated implants in the treatment of partially edentulous patients: a preliminary study on 876 consecutively placed fixtures. Int J Oral Maxillofac Implants. 1989;4:211-7.

2. Esposito M, Hirsch JM, Lekholm U, Thomsen P. Biological factors contributing to failures of osseointegrated oral implants. (II). Etiopathogenesis. Eur J Oral Sci. 1998;106:721-64.

3. Jemt T, Lekholm U. Implant treatment in edentulous maxillae: a 5 -year follow-up report on patients with different degrees of jaw resorption. Int J Oral Maxillofac Implants. 1995;10:303-11.

4. Molly L. Bone density and primary stability in implant therapy. Clin Oral Implants Res. 2006;17:124-35.

5. Martinez H, Davarpanah M, Missika P, Celletti R, Lazzara R. Optimal implant stabilization in low density bone. Clin Oral Implants Res. 2001;12:423-32.

6. Norton MR, Gamble C. Bone classification: an objective scale of bone density using the computerized tomography scan. Clin Oral Implants Res. 2001;12:79-84.

7. Turkyilmaz I, Tözüm TF, Tumer C. Bone density assessments of oral implant sites using computerized tomography. J Oral Rehabil. 2007;34:267-72.

8. Morris HF, Ochi S, Orenstein IH, Petrazzuolo V. AICRG, Part V: Factors influencing implant stability at placement and their influence on survival of Ankylos implants. J Oral Implantol. 2004;30:162-70. 9. Beer A, Gahleitner A, Holm A, Tschabitscher M, Homolka P. Correlation of insertion torques with bone mineral density from dental quantitative $\mathrm{CT}$ in the mandible. Clin Oral Implants Res. 2003;14:616-20.

10. Turkyilmaz I, Tumer C, Ozbek EN, Tözüm TF. Relations between the bone density values from computerized tomography, and implant stability parameters: a clinical study of 230 regular platform implants. J Clin Periodontol. 2007;34:716-22.

11. Turkyilmaz I, Tözüm TF, Tumer C, Ozbek EN. Assessment of correlation between computerized tomography values of the bone, and maximum torque and resonance frequency values at dental implant placement. J Oral Rehabil. 2006;33:881-8.

12. Ikumi N, Tsutsumi S. Assessment of correlation between computerized tomography values of the bone and cutting torque values at implant placement: a clinical study. Int J Oral Maxillofac Implants. 2005;20:253-60.

13. Friberg B, Jisander S, Widmark G, Lundgren A, Ivanoff CJ, Sennerby $\mathrm{L}$, et al. One-year prospective three-center study comparing the outcome of a "soft bone implant" (prototype Mk IV) and the standard Brånemark implant. Clin Implant Dent Relat Res. 2003;5:71-7. 14. Da Cunha HA, Francischone CE, Filho HN, De Oliveira RC. A comparison between 
cutting torque and resonance frequency in the assessment of primary stability and final torque capacity of standard and TiUnite singletooth implants under immediate loading. Int J Oral Maxillofac Implants. 2004;19:578-85.

15. Huang HM, Lee SY, Yeh CY, Lin CT. Resonance frequency assessment of dental implant stability with various bone qualities: a numerical approach. Clin Oral Implants Res. 2002;13:65-74.

16. Friberg B, Sennerby L, Linden B, Gröndahl K, Lekholm U. Stability measurements of one-stage Brånemark implants during healing in mandibles. A clinical resonance frequency analysis study. Int J Oral Maxillofac Surg. 1999;28:266-72.

17. Shahlaie M, Gantes B, Schulz E, Riggs M, Crigger M. Bone density assessments of dental implant sites: 1 . Quantitative computed tomography. Int J Oral Maxillofac Implants. 2003;18:224-31.

18. Aranyarachkul P, Caruso J, Gantes B, Schulz E, Riggs M, Dus I, et al. Bone density assessments of dental implant sites: 2 . Quantitative cone-beam computerized tomography. Int J Oral Maxillofac Implants. 2005;20:416-24.

19. Trisi P, Rao W. Bone classification: clinical-histomorphometric comparison. Clin Oral Implants Res. 1999;10:1-7. 